\section{Claude Férec}

Bernard Mercier Marie-Pierre Audrézet

\section{ADRESSE}

C. Férec: docteur en médecine, docteur ès sciences, directeur du centre de biogénétique, CDTS Brest. B. Mercier : docteur ès sciences, centre de biogénétique, CDTS Brest. M.P. Audrézet : doctorante, centre de biogénétique, CDTS Brest. Centre de biogénétique, CDTS, 46, rue Félix-Le-Dantec, 29275 Brest Cedex, France.

\title{
Les mutations de la mucoviscidose: du génotype au phénotype
}

Plus de quatre cents mutations ont été décrites dans le gène CFTR (cystic fibrosis transmembrane conductance regulator), responsables de la mucoviscidose ou fibrose kystique du pancréas. La caractérisation de ces diverses mutations du gène a permis une meilleure compréhension des relations structure/activité de la protéine CFTR. Certains traits cliniques de la maladie, telle la suffisance pancréatique, sont génétiquement déterminés. La compréhension de ces anomalies moléculaires laisse entrevoir des stratégies thérapeutiques différentes selon le type de mutation observée chez le patient.

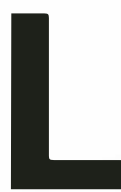

a mucoviscidose ou fibrose kystique du pancréas est aujourd'hui l'un des paradigmes de la génétique moléculaire. Illustration exemplaire de la puissance des technologies d'analyse de l'ADN puisque, dès 1985 , le gène était localisé sur le bras long du chromosome 7 en 7 q3.1 par une approche de génétique inverse appelée aujourd'hui clonage par positionnement $\left(\mathrm{m} / \mathrm{s} n^{\circ} 6\right.$, vol. 3, p. 369, [1]). Après quatre ans de marches et de sauts successifs dans cette région du chromosome 7 , le gène CFTR était cloné et séquencé en septembre 1989 par les équipes de Tsui, Collins et Riordan ([2-4], $m / s n^{\circ} 8$, vol. 5, p. 589). La réussite, en 1992, de l'invalidation du gène CFTR chez la souris a permis de développer un modèle animal inexistant préalable- ment $\left(\mathrm{m} / \mathrm{s} n^{\circ} 7\right.$, vol. 8, p. 653, [5]). Dans le même temps étaient créés de nouveaux vecteurs viraux [6] pour le transfert de gène qui allaient conduire au cours de ces derniers mois aux premiers essais de thérapie génique chez l'homme à l'aide d'adénovirus recombinants. C'est dire le chemin parcouru depuis que le gène responsable a été cloné et séquencé en 1989. Ce gène, composé de 27 exons, s'étend sur $230000 \mathrm{pb}$ et code pour une protéine de 1480 acides aminés appelée CFTR pour cystic fibrosis transmembrane conductance regulator. Dès sa découverte, une délétion de trois paires de bases située dans l'exon 10 fut mise en évidence, délétion correspondant dans la protéine mutée à la perte d'une phénylalanine en position $508(\triangle \mathrm{F} 508)$.

Aujourd'hui, la connaissance des 


\section{RÉFÉRENCES}

1. Tsui LC, Buchwald M, Barker D, el al Cystic fibrosis locus defined by a genetically polymorphic DNA marker. Science 1985; 230: 1054-7.

2. Kerem BS, Rommens JM, Buchanan JA, et al. Identification of the cystic fibrosis gene: genetic analysis. Science 1989 ; 245 1073-80.

3. Riordan JR, Rommens JM, Kerem BS, et al. Identification of the cystic fibrosis gene : cloning and characterization of complementary DNA. Science 1989; 245 : 1066-73.

4. Rommens JM, Iannuzi MC; Kerem BS, et al. Identification of the cystic fibrosis gene: chromosome walking and jumping. Science 1989 ; 245 : 1059-64.

5. Dorin JR, Dickinson P, Alton EWFW Smith SN, Geddes DM, Stevenson BJ, Kimber WL, Fleming S, (larke AR, Hooper ML, Anderson L, Beddington RSP, Porteous DJ. Cystic fibrosis in the mouse by targeted insertional mutagenesis. Nalure 1992 ; 359 : $211-5$.

6. Rosenf eld MA, el al. In vivo transfer of the human cystic fibrosis transmembrane conductance regulator gene to the airway epithelium. Cell 1992 ; 68 : 143-55.

7. Tsui LC. Mutations and sequence variations detected in the cystic fibrosis transmembrane conductance regulator (CFTR) gene: a report from the (.ystic Fibrosis Genetic Analysis Consortium. Human Mulıtion 1992; 1: 197-203.

8. Worldwide survey of the delta F508 mutation report from the Cystic Fibrosis Genetic Analysis Consortium. Am J Hum Genet 1990; 47 : 354-9.

9. Serre JL, Simon-Bouy B, Mornet E, Jaume-Roig B, Balassopoulou A, Schwarty M, Taillandier A, Boué J, Boué A. Studies of RFLP closely linked to the cystic fibrosis locus throughout Europe lead to new considerations in population genetics. Hum Genet 1990 ; 84 : 449-54.

10. EWCG: gradient of distribution in Europe of the major (.F mutation and of its associated haplotype. Hum Genet 1990 ;

anomalies moléculaires du gène ( $F^{2} T R$ s'est enrichie de façon inattendue et spectaculaire puisque plus de quatre cents mutations ont été décrites en quatre ans (Cystic jibrosis genetic analysis consortium, communication personnelle). De l'apparente complexité de cette pathologie moléculaire, il est possible de dégager des enseignements concemant: (1) la distribution des mutations dans le gène CFTR, (2) la nature des anomalies moléculaires, (3) les relations génotype/phénotype cliez les patients atteints de mucoviscidose, (4) les applications diagnostiques apportées par la commaissance des mutations du gène.

\section{Spectre de distribution des mutations dans le gène CFTR}

Plus de 20000) chromosomes CF (provenant de patients atteints de mucoviscidose) ont été analysés par la centaine de laboratoires réunis au sein d'un Consortium international d'étude des mutations du gène coordommé par lap Chee Tsui à Toronto. La mutation $\Delta \mathrm{F} .008$ a été retrouvée associée globalement à $68 \%$ de ces cliromosomes [7]. Cette valeur moyenne recourre des disparités importantes de répartition liées à l'origine ethnique et géographique des patients $\left(\mathrm{m} / \mathrm{s} n^{\circ} 1\right.$, vol. $8, p$ ) 86). Ainsi, la mutation $\Delta \mathrm{F} 508$ est-elle associée à $88 \%$ des chromosomes mutés au Danemark et à 30\% seulement en Turquie [8]. Cette mutation $\Delta \mathrm{F} 508$ est présente presque exclusivement au sein de la population blanche d'origine européenne, ce qui explique pour une grande part l'incidence élevée de la maladie, proche de 1 sur 2500 , chez les Européens et leurs descendants aux États-Unis et au Canada. Il avait été montré, peu de temps après la découverte du gène, qu'il existait en Europe un gradient Nord Ouest/Sud Est dans la distribution de la mutation $\Delta \mathrm{F} 5(08$. Un groupe de travail européen consacré à la génétique de la mucoviscidose avait émis l'hypothèse, pour expliquer cette distribution, que la mutation $\Delta F 5(08$ se serait disséminée en Europe au cours des migrations néolithiques de fermiers venus de l'est et du centre du continent $[9,10]$. En fait, les études du gène réalisées chez les Basques - la plus vieille population européennc qui s'est étatblie au Paléolithique — montrent sans ambiguité que la mutation $\Delta$ F508 était déjà présente dans cette population. Cela vient d'être confirmé par un travail européen mené à l'initiative du groupe d'Estivill à Barcelone (Espagne) [11]. L'étude des marqueurs intra-géniques très polymorphes de type microsatellite a permis d'étudier la distribution des haplotypes associés à la mutation $\Delta$ F50) au travers d'un échantillon significatif de 1738 chromosomes provenant de quinze régions européennes. Les résultats obtenus confirment l'hypothèse précédemment avancée d'un chromosome ancestral portant la mutation $\Delta \mathrm{F} 508$ [12]. C.e travail permet de situer à une période de 65000 à 200000 ans l'apparition probable de la mutation $\Delta$ F508 [13]. Aujourd'hui, la distribution observée de cette mutation serait le reflet des vagues successives de peuplement ayant contribué à la constitution des différentes populations de l'Europe actuelle.

La fréquence de la mutation $\Delta \mathrm{F} 508$ est de $70 \%$ en France [14]; cette valeur moyemne masque des variations importantes allant de $81 \%$ en Bretagne occidentale, à $64 \%$ en Languedoc-Roussillon ou $65 \%$ en Normandie [15-17].

Parmi les quatre cents mutations rapportées au travers du Consortium international, seul un petit nombre dépasse le seuil de $1 \%$ dans les différentes populations de patients étudiés. Il s'agit des mutations (3542X $(3.4 \%), \quad$ G551D (2.4\%), W1282X (2.1\%), N1303K (1.8\%), R553X $(1.3 \%), 621+1 \mathrm{G} \rightarrow \mathrm{T}(1.3 \%)$, $1717-1 \mathrm{G} \rightarrow \mathrm{A}(1.1 \%)$ (Cystic fibrosis genetic analysis consortium, communication personnelle). Il est important de souligner que le spectre de distribution des mutations du gène C $F^{2} T R$ est réalisé à partir de la compilation de dommées obtenues par les cent laboratoires du Consortium et ne reflète en aucun cas leur distribution géographique [18].

Ces mutations peu fréquentes du gène $\left(F^{2} T R\right.$ sont le reflet de l'origine ethnique des patients. La fréquence de la mutation (5551D est d'envi- 
ron $5 \%$ dans les populations d'origine celte (Irlande, Écosse, Bretagne, centre de l'Europe, Tchécoslovaquie) [19]. Ia mutation (6542X est relativement fréquente dans les pays du pourtour méditerranéen [20], la mutation W'1282X est associée à 60) \% des chromosomes CF dans la population juive ashkénaze [21]. Quelques équipes ont pu mener à bien l'étude complète des 27 exons du gène et de leur région flanquante (jonction intron/exon). Ainsi, en Bretagne, dix-neuf mutations différentes rendent compte de
$98 \%$ des mutations du gène, des résultats comparables ont été retrouvés au Québec et au Pays de Galles $[15,22,23]$. A l'inverse, dans les grandes métropoles où le brassage des populations a été plus important, l'analyse exhaustive des régions codantes du gène a permis d'identifier $88 \%$ des anomalies moléculaires à Paris [24] et $91 \%$ à Montpellier [25]. La figure 1 illustre cette diversité dans la distribution des fréquences des cinq principales mutations de quelques régions françaises. En Europe centrale, il existe assez

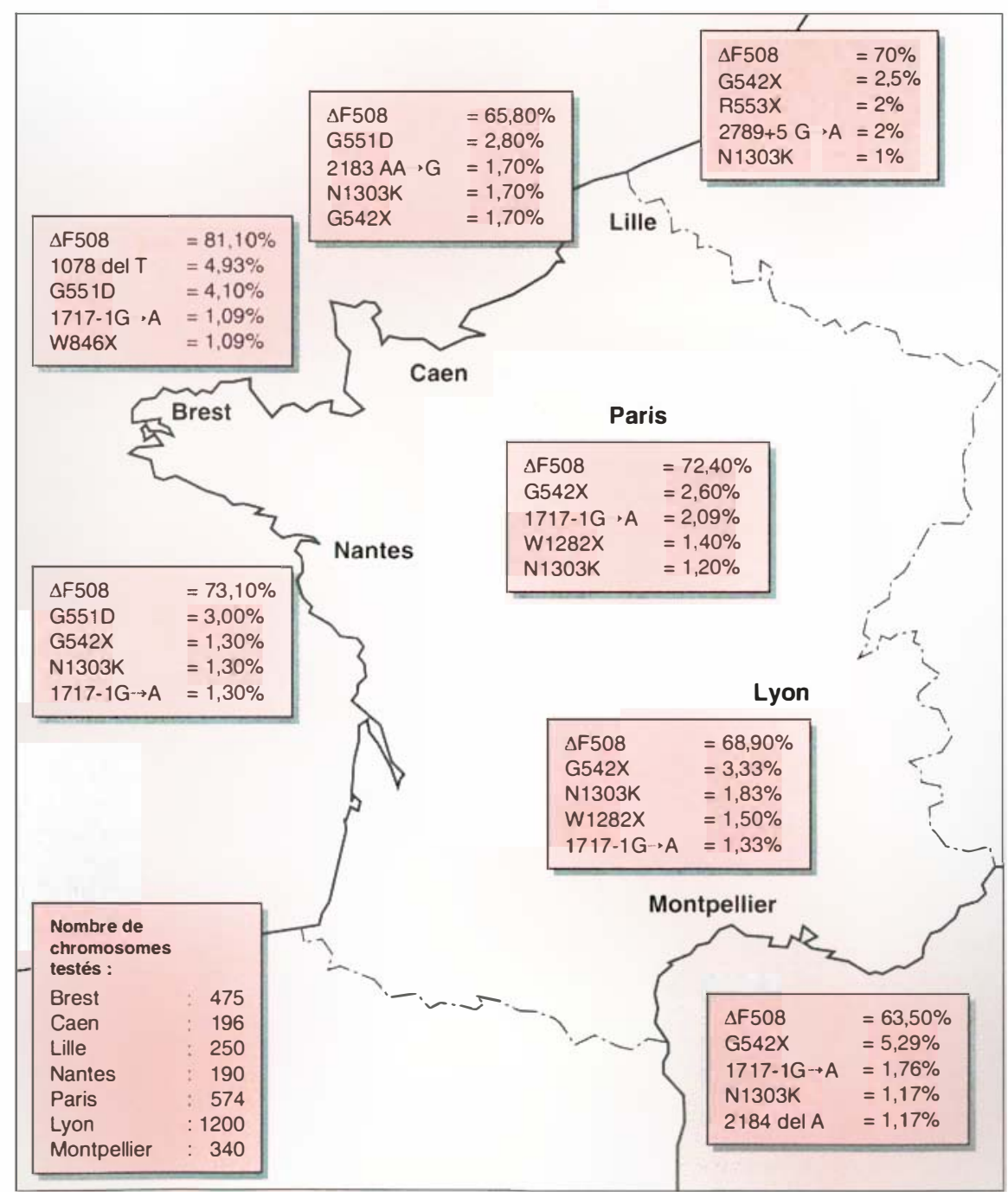

Figure 1. Diversité dans la distribution des fréquences des principales mutations de quelques régions françaises. Les acides aminés sont dénommés par le code à une lettre: A: Ala; C: Cys; D: Asp; $E: G l u ; F$ : Phe; G: Gly; H: His; I: Ile; K: Lys; L: Leu; M: Met; N: Asn; P: Pro; $O:$ Gln; R: Arg; $S:$ Ser; T: Thr; V: Val; W: Trp; Y:Tyr. peu d'études systématiques des mutations du gène $C F T R$; nous avons récemment étudié des séries de patients originaires de Slovénie et de Bulgarie [26, 27] et montré qu'il n'existait pas d'effet fondateur particulier dans ces pays. L'étude complète du gène a permis d'identifier $84 \%$ des mutations en Slovénie et $90 \%$ en Bulgarie.

La situation reste particulière au sud de l'Europe ; ainsi, en Espagne et en Italie, 30\% des mutations sont non identifiées et très probablement situées en dehors de la séquence codante du gène.

\section{Nature des anomalies moléculaires du gène}

Le gène CHTR code pour une protéine transmembranaire qui est un canal chlore dont l'activité est réglée par l'ATP cytosolique et par des phosphorylations. Les deux sites de fixation de l'ATP (NBF pour nucleotide binding fold) jouent un rôle fondamental en interagissant directement avec I'ATP pour l'ouverture du canal. Le domaine $\mathrm{R}$ (regulatory domain) présente 4 sérines qui sont phosphorylées in vivo après activation de la protéine kinase $\mathrm{A}$ (PKA) [28]. II semble que le domaine $\mathrm{R}$ inhibe le passage des ions au travers du canal lorsqu'il est non phosphorylé, et que deux étapes soient nécessaires pour le bon fonctionnement du canal chlore. La première étape implique la phosphorylation du domaine $\mathrm{R}$ après stimulation de la PKA par l'AMPc; au cours de la seconde étape, cette forme phosphorylée du CFTR se lie à l'ATP et une modification conformationnelle de la protéine intervient, favorisant le passage passif des ions chlorures. Les ions chlorures traversent la membrane selon un gradient électrochimique. Ce n'est là qu'un modèle, proposé aujourd'hui, mais rendant assez bien compte des faits expérimentaux $\left(m / s n^{\circ} 8\right.$, vol. 5, p. 872, [29, 30]) (figure 2).

Le gène CFTR est le siège de nombreuses mutations ponctuelles ou de très courtes insertions-délétions. Les grandes délétions sont, en revanche, peu fréquentes; seules quelques descriptions de chromosomes portant 


\section{RÉFÉRENCES}

11. Morral N, Bertran petit J, Estivill X,et al. Tracing the origin of the major cystic fibrosis mutation $(\Delta \mathrm{F} 508)$ in European populations. Nature Genet 1994 (sous presse).

12. Estivill X, Scambler PJ, Wainwright BJ. Patterns of polymorphism and linkage disequilibrium for cystic fibrosis. Genomics $1987 ; 1: 257-63$.

13. Morral N, Nunes V, Casals T, Chillon M, Giménez J, Bertranpetit J, Estivill X Microsatellite haplotypes for cystic fibrosis: mutation frameworks and evolutionary tracers. Hum Mol Genet 1993; 2: 1015-22.

14. Vidaud M, Férec C, Attree O, et al. Frequency of the cystic fibrosis delta F508 mutation in a large sample of the French population. Hum Genet 1990 ; 85 : 434-5.

15. Férec C, Audrézet MP, Mercier B, et al. Detection of over $98 \%$ cystic fibrosis mutations in a Celtic population. Nature Genet 1992; 1 : 188-91.

16. Claustres M, Desgeorges M, Kjellberg P, Bellet H, Demaille J, Ramsay M. Cystic fibrosis typing with DNA probes and screening for Delta F508 deletion in families from Southern France. Hum Genet 1990 ; 85 : 398-9.

17. Verlingue C, Mercier B, Lecoq I, Audrézet MP, Laroche D, Travert G, Férec C. Retrospective study of the CFTR gene mutations in Guthrie cards from a large cohort of neonatal screening for cystic fibrosis. Hum Genet 1993 (sous presse).

18. Serre JL, Mornet E, Simon-Bouy B, Boué J, Boué A. General cystic fibrosis mutations are usually missense mutations affecting two specific protein domains and associated with a specific RFLP marker haplotype. Eur J Hum Genet 1993; 1: 287-95.

19. Hamosh A, King TM, Rosenstein BJ, et al. Cystic fibrosis patients bearing both the common missense mutation Gly $\rightarrow$ Asp at codon 551 and the F508 mutation are clinically indistinguishable from F508 homozygotes, except for decreased risk of meconium ileus. Am J Hum Genet 1992; $51: 245-50$

20. Nunes V, Gasparini P, Novelli G, et al. Analysis of 14 cystic fibrosis mutations in five South European populations. Hum Genet 1991; 87 : 737-8

21. Shoshani T, Augarten A, Gazit E, et al. Association of a nonsense mutation (W1282X), the most common mutation in the Ashkenazi Jewish cystic fibrosis patients in Israel, with presentation of severe

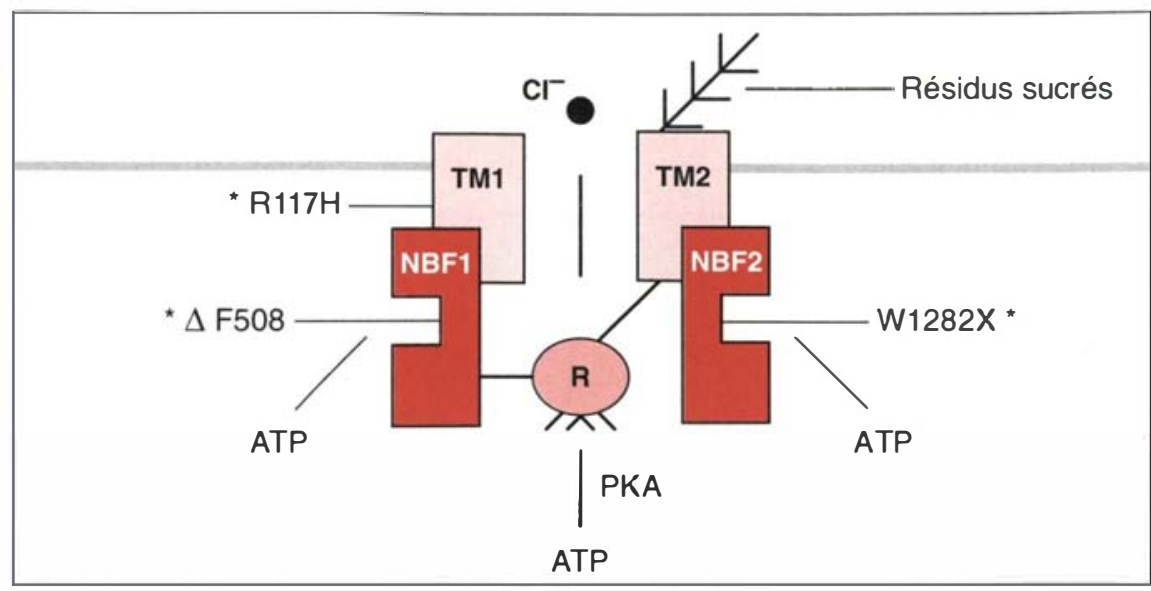

Figure 2. Structure schématisée de la protéine CFTR. TM1 et TM2 représentent les domaines transmembranaires amino- et carboxy-terminaux, NBF1 et NBF2 les sites de liaison de l'ATP (nucleotide binding fold), $R$ le domaine régulateur, phosphorylé par la protéine kinase dépendante de l'AMPC (PKA). Trois sites de mutations sont marqués sur la figure par un astérisque.

Figure 3. Répartition des anomalies moléculaires dans le gène.
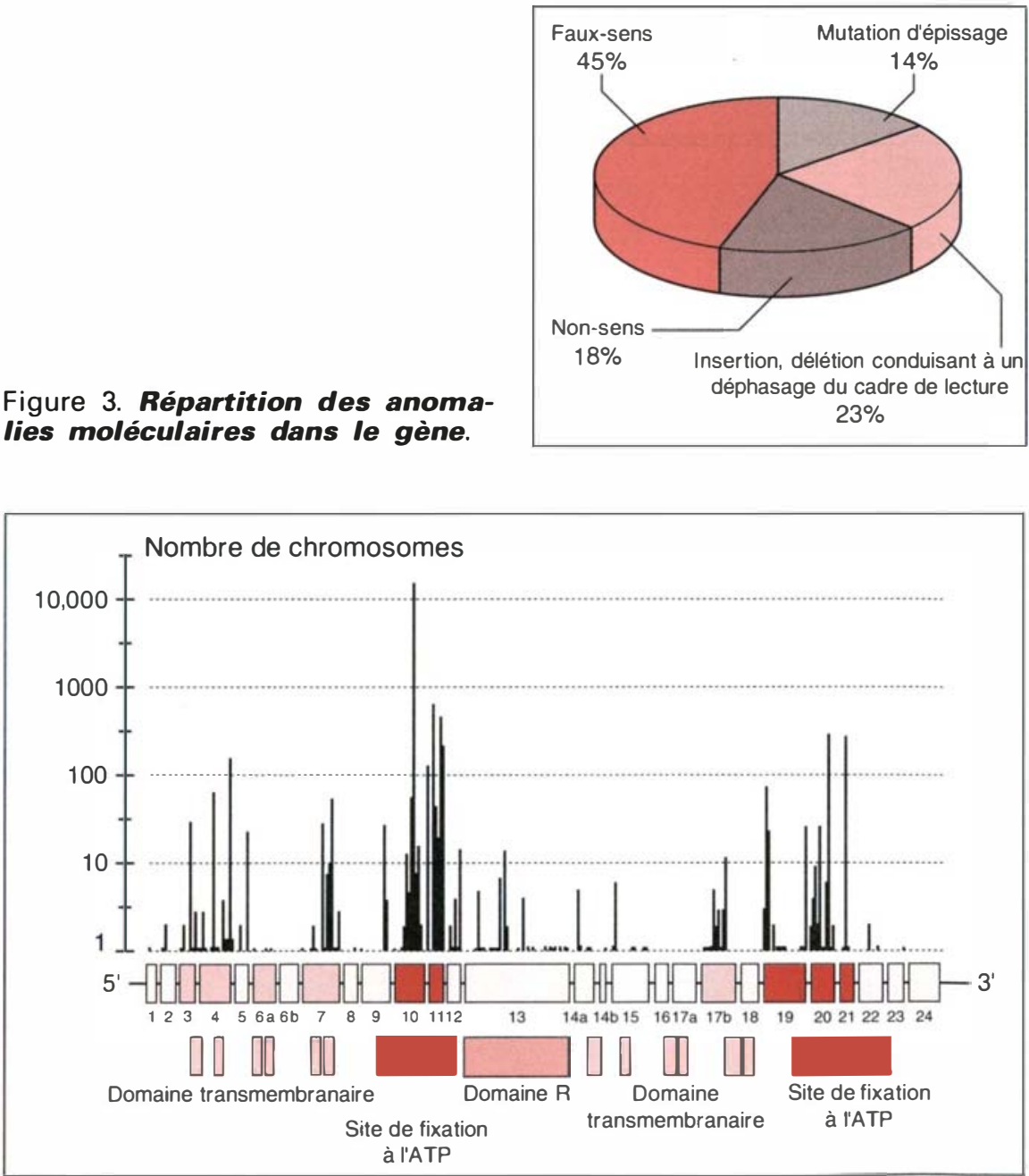

Figure 4. Localisations et fréquences des mutations dans le gène CFTR. Source: L.C. Tsui. Consortium international d'étude des mutations du gène CFTR [57]. 
des délétions de $50 \mathrm{~kb}$ ont été rapportées [31].

L'essentiel des mutations se répartit en mutations faux sens $(45 \%)$, non sens $(18 \%)$, insertions-délétions conduisant à un déphasage du cadre de lecture $(23 \%)$ et des mutations d'épissage $(14 \%)$ (figure 3). La dispersion des mutations dans le gène n'est pas homogène, avec des exons qui semblent, plus fréquemment que d'autres, être le siège de mutations ponctuelles (en particulier ceux codant pour les deux sites de liaison à l'ATP, NBF) (figures 4, 5). C'est le cas des exons 10 et $11,19,20$ et 21 codant respectivement pour le premier et le second NBF. A l'heure actuelle, les mutations situées dans les exons codant pour les régions transmembranaires du gène, en particulier les exons 3, 4 et $17 \mathrm{~b}$, sont aussi le siège de nombreuses mutations bien que leur fréquence respective soit peu élevée.

L'étude systématique des messagers du gène CFTR a montré, chez les sujets normaux, de très fréquents épissages alternatifs se traduisant par la présence d'allèles dont le transcrit peut être délété d'un ou plusieurs exons. Ces épissages alternatifs mettent fréquemment en jeu l'exon 9 du gène. Chu et al. ont en effet montré que le transcrit exon 9 pouvait représenter jusqu'à $90 \%$ des messagers chez un sujet normal [32]. Cet épissage alternatif incomplet est dû à la présence d'un polymorphisme de séquence polypyrimidique (suite de 5T, 7T ou 9T), situé dans le site accepteur de l'intron 8. Ainsi, une séquence de 5T conduit à un épissage très incomplet.

Ces anomalies d'épissage du gène peuvent, très probablement, rendre compte des variations phénotypiques importantes observées chez des patients porteurs de mêmes mutations. Cela a été montré récemment pour la mutation R117H [33]. Les auteurs ont montré que cette mutation pouvait être une mutation récurrente portée par des haplotypes différents. L'un des haplotypes, portant un polymorphisme de répétition de 5T dans l'intron 8, conduit à un épissage incomplet; dans l'autre haplotype, un polymorphisme de $7 \mathrm{~T}$ induit un épissage correct. Le premier haplotype est retrouvé en majorité chez des malades présentant une forme clinique de mucoviscidose avec suffisance pancréatique, le second est retrouvé uniquement chez des patients présentant une agénésie des canaux déférents. Cela souligne bien la complexité des corrélations génotype/phénotype lorsque la même mutation est portée par des haplotypes différents. Il a

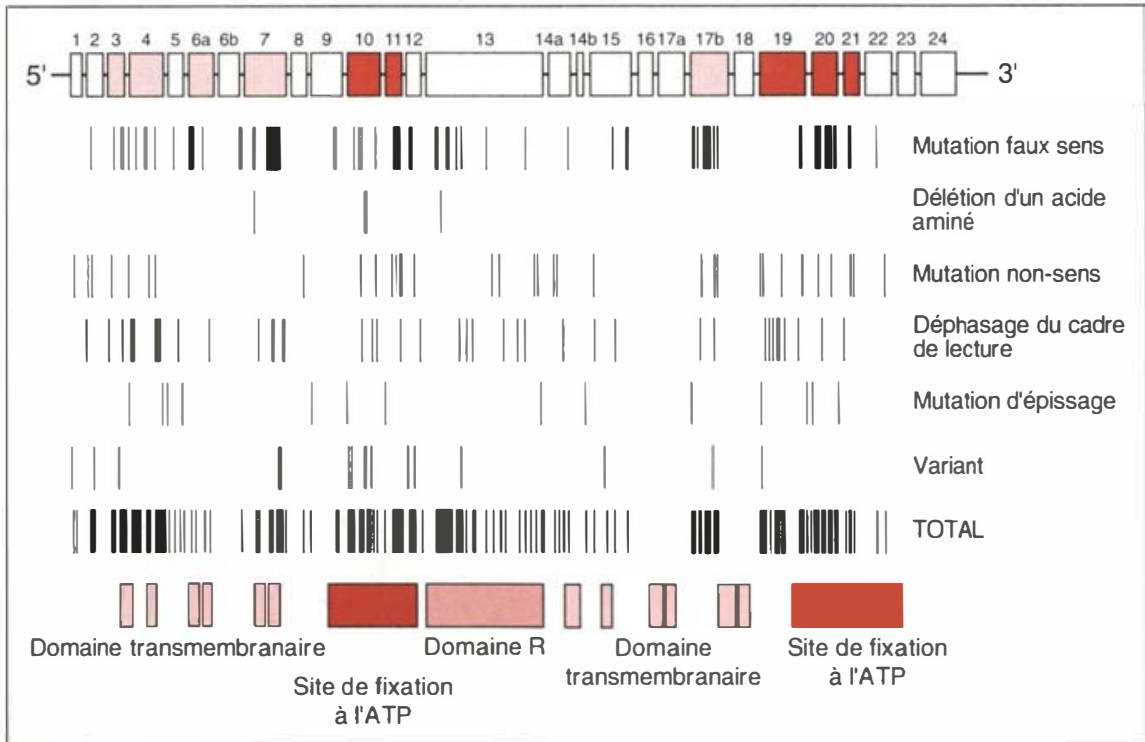

Figure 5. Distribution des mutations dans le gène CFTR. Source: L.C. Tsui. Consortium international d'étude des mutations du gène CFTR [58]. également été montré que l'association de plusieurs mutations sur le même allèle pouvait conduire à des réversions du phénotype, et qu'une seconde mutation pouvait moduler l'effet de la première [34].

Afin d'analyser et de tirer des enseignements de cette très riche pathologie moléculaire, Welsh et Smith ont récemment proposé une répartition des mutations en quatre classes en fonction du type d'anomalie moléculaire [35] (figure 6).

Classe 1. Les mutations affectant la production de la protéine

Elles se traduisent par une absence totale ou partielle de la protéine. Sont inclues dans cette catégorie: les mutations non sens, les mutations d'épissage, les mutations entraînant un déphasage du cadre de lecture. La mutation W1282X, fréquente chez les malades d'origine juive ashkénaze, est un bon exemple de ce type de mutation. Cette mutation ponctuelle en position $3978, \mathrm{G} \rightarrow \mathrm{A}$, conduit au changement du codon TGG (Tryptophane $=\mathrm{W}$ ) en TGA (codon stop).

Classe 2. Les mutations affectant la maturation cellulaire de la protéine CFTR, telles la $\Delta \mathrm{F} 508$ ou la $\Delta \mathrm{I} 507$ où des anomalies de la glycosylation conduisent à une mauvaise circulation intracellulaire et à un mauvais positionnement de la protéine dans la partie apicale de la cellule épithéliale pulmonaire.

\section{Classe 3. Les mutations affectant la régulation du canal chlore}

Il s'agit de mutations faux sens, situées au niveau des sites de liaison de l'ATP (NBF I ou II). Ces mutations entraînent une altération de la fonction du CFTR qui est réglé par la concentration en ATP cytosolique. Un exemple de mutation conduisant à de telles dérégulations du canal chlore est la mutation G551D, provoquant au sein du premier NBF le remplacement d'une glycine en acide aspartique.

Classe 4. Les mutations produisant un dysfonctionnement de la conduction

Les régions transmembranaires de la 


\section{RÉFÉRENCES}

22. Rozen R, Schwarty. RH, Hilman BC; et al. Cystic fibrosis mutations in North American populations of French ancestry : analysis of Quebec French-Canadian and Louisiana Acadian families. Am I Hum Genet 1990); $47: 6(06 \cdot 10$

23. Cheadle JP, Goodchild MC, Meredith AL. Direct sequencing of the complete C.FTR gene: the molecular characterisation of $99.5 \%$ of C.F chromosomes in W'ales. Hum Mol Cienet 1993; 2, 10: 1551-6.

24. Fanen P, Ghanem N, Viclaud M, Besmond (;, Martin J, Costes P, Plassa F, (joossens M. Molecular characterization of cystic fibrosis: 16 novel mutations identified by analysis of the whole cystic fibrosis transmembrane conductance regulator (C.FTR) coding regions and splice site junctions. Genomics 1992; 13: 770-6.

25. Claustres M, Laussel M, Desgeorges $\mathbf{M}$, Giansily M, Culard JF, Razakatsara (;, Demaille J. Analysis of the 27 exons and flanking regions of the cystic fibrosis gene: $4 *$ different mutations account for $91.2 \%$ of the mutant alleles in Southern France. Hum Mol Genet 1993; 2: 1209-13.

26. Savov A, Mercier B., Kalaydjieva I., Férec C. Identification of six novel mutations in the C:FTR gene of patients from Bulgaria by screening the twenty seven exons and exon/intron boundaries using I)G( $; E$ and direct I)NA sequencing. Hum Mol Cienetirs 1994; 3: 57-60.

27. Audrézet MP, Canki Klain N, Mercier B, Bravar I), V'erlingue (', Férec C. Identification of three novel mutations in the Slovenian population. Hum (ienet 1994 (sous presse)

28. ('heng SH, Rich IDP, Marshall J, (iregory RJ, W'elsh MJ, Smith AE. Phosphorylation in the $R$ domain by cAMP. dependant protein kinase regulates the CFTR chloride channel. Cell 1991; 66 1027-36.

29. Collins FS. Cystic fibrosis: molecular biology and therapeutic implications. Srience 1992 ; 256: 774-9.

30. (joossens M. Biologie de la mucoviscidose: progrès récents et perspectives. médecine/sciences 1991; $7: 1048-51$

31. Morral N, Nunes V', Casals T, Cobos N, Asensio (), Dapena J, Estivill X. Uniparental inheritance of microsatellite alleles of the cystic fibrosis gene (C.FTR) : identification of a 5() kilobase deletion. Hum $\mathrm{Mol}$ Genet 1993; 2, 6: 677-81.

32. Chu SC, Trapnel BC, Curristin S, Cutting CR, C.rystal RG. Cenetic basis of variable exon 9 skipping in cystic fibrosis transmembrane conductance regulator mRNA. Nature Cienet 1993; 3: 151-6.

33. Kiesewetter S, Macek M Jr, I)avis (;, Curristin SM, Chu CS, Graham C, Shrimp- ton AE, Cashman SM, Tsui I.C, Mickle J, Amos J, Highsmith UE, Shuber A, W'it I)R, Cirystal R(;, Cutting GR. A mutation in C.FT'R produces different phenotypes depending on chromosomal background. Nature Genet 1993; 5 : 274-8.

34. Teem JI, Berger HA, Ostedgaard LS, Rich I)P, Tsui L.(;, W'elsh MJ. Identification of revertants for the cystic fibrosis F5()8 mutation using STE6-CFTR chimeras in yeast. Cell $1993 ; 73$ : 335-46.

35. W'elsh MI, Smith AE. Molecular mechanisms of (.FTR chloride channe dysfunction in cystic fibrosis. (ell 1993; 73 1251-4.

36. Sheppard IDN, Rich DP, ()stedgaard IS, Gregorv RJ, Smith AE, Welsh M. Mutations in CFTR associated with mild-cliseaseform (it- channels with altered pore properties. Nature 1993; 362: 160)-4.

37. Boat TF, Welsh MJ, Beaudet AL. The Metabolic basis of inherited disease. In : Scriver (.R, Beaudet AL., Sly WS, V'alle I), eds. New York: Mc( Graw Hill, 1989: 2649-80.

38. Kerem E, (iorey M, Kerem BS, et al. The relation between genotype and phenotype in cystic fibrosis: analvsis of the most common mutation (F5()8). $N$ lingl Ifed 1990) 323: 1517-22.

39. Santis (;, Osborne I, Knight RA, Hodson ME. Inclependant genetic determinant. of pancreatic and pulmonasy status in cystic fibrosis. l.ancel 1990) 336i: 1081-4.

40. Santis (;, ()sborne L, Knight RA, Hodson ME. I.inked marker haplotypes and the F5()8 mutation in adults with mild pulmonary disease and custic fibrosis. Lancet 1990 ; 335 : 1426-9.

41. Kristidis P, Bozon D, Corey M, Markiewicz D, Rommens J, Tsui I.C., Durie P. (ienetic determination of exocrine pancreatic function in cystic fibrosis. A $\mathrm{m}$ / Hum (ienet 1992; 50): 1178-84.

42. Guillermit H, Jéhanne M, Quéré I Auclézet MP, Mercier B, Férec C. A novel mutation in exon 3 of the (FTR gene. Hum Cienet 1993; 91 : 233-5.

43. Mercier B, I issens W, Novelli (;, el al Identification of cight novel mutations in a collaborative analysis of a part of the second transmembrane domain of the CFTR gene. Cienomics 1993; 16: 296-7.

44. Férec C:, V'erlingue C, Mercier B, (juillermit H, Quéré I, Raguénès (), Audrézet MP. Genotvpe analysis of adult cystic fibro sis patients. Hum Mol Genet 1993; 2, I () : $1557-60$.

45. Tabcharani JA, Rommens JM, Hou IX Chang XB, Tsui I.C, Riordan JR, Hanrahan JW. Multi-ion pore behaviour in the (FTR chloride channel. Nature 1993; 366 79-82. protéine constituent un canal ayant un rôle important dans la conduction et la sélectivité ionique. Il est maintenant établi que les mutations faux sens situées dans ces régions transmembranaires affectent la sélectivité du canal chlore. Il a été montré récemment par Sheppard que ce type de mutant C.FTR transfecté en système cellulaire hétérologue produit un CFTR correctement positionné et contrôlé par l'AMPc mais dont la conductance est diminuée [36]. Le prototype de ces mutations est la R117H ou la R334W.

\section{Corrélation}

\section{génotype/phénotype}

La diversité des signes cliniques de la mucoviscidose et les profils évolutifs très différents d'un patient à l'autre ont naturellement conduit à rechercher des corrélations entre le génotype et le phénotype des patients $\left(\mathrm{m} / \mathrm{s} n^{\circ} 12\right.$, vol. 9, p. 1431). Il avait été noté depuis longtemps que $10 \%$ à $15 \%$ d'entre eux présentaient une fonction pancréatique externe conservéc et ces patients étaient qualifiés de "pancréatiques suffisants " [37].

La connaissance de la mutation principale, la $\Delta$ F508 en 1989-1990, permit dès cette époque de montrer la relative homogénéité clinique des patients homozygotes pour cette mutation [38]. Ceux-ci avaient, dans leur très grande majorité, une forme classique de la maladie avec atteinte pulmonaire importante et insuffisance pancréatique. Une différence apparaissait dans la présentation clinique des malades homozygotes $\triangle F 508$ et des sujets hétérozygotes composites (porteurs d'une ou de deux mutations non identifiées) [39, 40]. Ces patients hétérozygotes composites avaient, en règle générale, une atteinte moins sévère de la fonction pulmonaire.

Un rôle important pour les mutations autres que la mutation $\Delta \mathrm{F} 508$ avait été proposé dès la découverte du gène par Lap Chee Tsui ; il avait estimé qu'au moins un facteur clinique, la suffisance pancréatique, était génétiquement déterminé, et que les chromosomes porteurs d'une mutation qualifiée de non sévère (mild) avaient un eff et domi- 


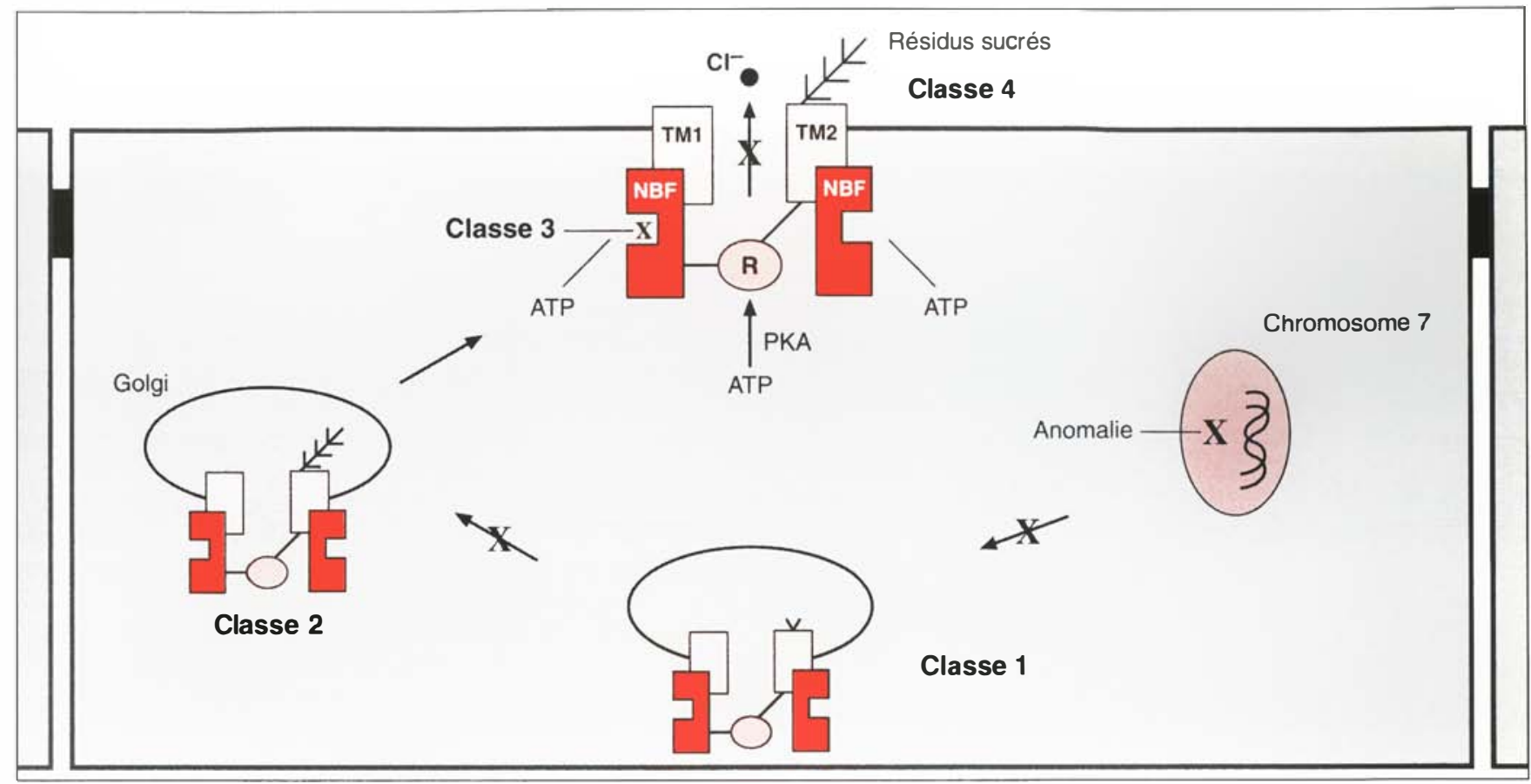

Figure 6. Structure et fonction du CFTR. Les mutations du CFTR ont été divisées en quatre classes. Classe 1 : mutations affectant la production de la protéine (déphasage du cadre de lecture, codon stop, mutation d'épissage); classe 2: mutations affectant la maturation de la protéine, les anomalies de glycosylation conduisent à une mauvaise circulation intracellulaire et à un mauvais positionnement de la protéine dans la partie apicale de la cellule épithéliale pulmonaire; classe 3: mutations affectant la régulation du canal chlorure par la concentration cellulaire d'ATP; classe 4: mutations affectant la sélectivité du canal ionique et entraînant des troubles de la conduction.

nant par rapport aux autres mutations qualifiées de sévères, la mutation $\Delta \mathrm{F} 5() 8$ en étant le chef de file. C.e modèle a été très largement confirmé par le groupe de Toronto [41] dès que l'identification des mutations rares a été plus complète. Nous avons également identifié quelques nouveaux allèles CFTR chez des patients sans insuffisance pancréatique (G91R, H1054D) [42, 43]. Par ailleurs, nous nous sommes intéressés à l'étude du génotype de patients âgés de plus de 35 ans porteurs d'une forme fruste de la maladic. Une exploration systématique et exhaustive de l'ensemble de la séquence codante du gène par la technique d'électrophorèse en gradient de gel dénaturant nous a permis d'identifier le génotype complet de sept patients. Il s'agit, pour l'essentiel, de mutations faux sens situées dans les domaines transmembranaires, conduisant à des troubles de la conduction du canal chlorure [44]. L’approche génétique qui a conduit à l'identification précise $\mathrm{m} / \mathrm{s} n^{\circ}$ 6-7 rol. 10, juin-juillet 94 de ces mutations associées à des formes frustes a été confirmée récemment par Sheppard [36] qui a construit des mutants C.FTR (R334W, $\mathrm{R} 347 \mathrm{H})$ par mutagenèse dirigée et les a fait s'exprimer dans un système cellulaire hétérologue; il a montré que cette protéine (.FTR mutée conservait environ $10 \%$ de conductance du chlore. L'exploration de ces mutations transmembranaires a été encore récemment complétée par Tabcharani. Par la même approche, il a montré que l'arginine en position 347 mutée en histidine, proline ou leucine conduisait à la modification de la fonction de la protéine CFTR, qui de canal multi-ionique devenait simplement capable d'assurer le passage d'un seul ion [45]. Ces résultats soulignent l'intérêt de la définition précise des génotypes des patients; cette connaissance pourrait, dans les prochaines années, être d'une importance capitale dans le choix, pour un malade donné, d'une thérapeutique spécifique de la mucoviscidose.

\section{Les formes particulières de la mucoviscidose}

\section{L'agénésie déférentielle}

La description de plus de quatre cents allèles (FTR mutés rend compte de l'hétérogénéité génotypique de la maladie et les formes frontières ou particulières de la maladie sont aujourd'hui réexaminées avec une attention toute particulière à la lumière des informations que nous apporte la connaissance du gène (FTR. Ainsi, en 1990, l'équipe de Roussel à Lille $\left(m / s n^{\circ} 3\right.$, vol. 9, p. 344 [46]) a-t-elle recherché systématiquement la présence d'éventuelles anomalies moléculaires du gène CFTR chez des patients suivis pour des problèmes de stérilité due à une agénésie des canaux déférents. On savait, en effet, depuis longtemps, d'une part, que les hommes atteints de mucoviscidose présentaient une stérilité excrétoire liée à une agénésic déférentielle, d'autre part, que cette forme de stérilité 


\section{RÉFÉRENCES}

46. Rigot JM, Lafitte IJ, Dumur V, Gervais $\mathrm{R}$, Manouvrier S, Biserte J, Mazeman E, Roussel P. Cystic fibrosis and congenital absence of the vas deferens. $N \mathrm{Engl} / \mathrm{Med}$ $1991 ; 325,1: 64-5$.

47. Gervais R, Dumur V, Rigot JM, Lafitte IJ, Roussel P, Claustres M, Demaille J. High frequency of the $\mathrm{R} 117 \mathrm{H}$ cystic fibrosis mutation in patients with congenital absence of the vas deferens. $N$ Engl J Med 1993 ; 328 : 447-57

48. Simon-Bouy B, Stern M, Taillandier A, Mornet E, Boué A, Caubarrère I. Increased frequency of the F508 mutation in patients with bronchiectasis. Pediatric Pulmonology, 1993, Seventh Annual North American Cystic Fibrosis Conference. Suppl. 9, 127.

49. Strong TV, Smit LS, Turpin SV, Cole JL, Tom Hon C, Markiewicz D, Petty TL, Craig MW, Rosenow EC, Tsui LC, Iannuzzi MC, Knowles MR, Collins FS. Cystic fibrosis gene mutation in two sisters with mild disease and normal sweat electrolyte levels. N Engl J Med 1991; 325 : 1630-4.

50. Augarten A, Kerem BS, Yahav Y, Noiman S, Rivlin Y, Tal A, Blau H, Ben-Tur L, Szeinberg A, Kerem E, Gazit E. Mild cystic fibrosis and normal or borderline sweat test in patients with the $3849+10 \mathrm{~kb}$ C.AET mutation. Lancet 1993; 342: 25-6.

51. Férec C, Verlingue C, Audrézet MP, et al. Prenatal diagnosis of cystic fibrosis in different European populations: application of denaturing gradient gel electrophoresis. Fetal Diagnosis and Therapy 1993; 8 : 341-50.

52. Morral N, Girvau E, Zielenski J, Nunes V, Casals T, Tsui LC, Estivill X. Dinucleotide (CA/GT) repeat polymorphism in intron $17 \mathrm{~b}$ of the cystic fibrosis transmembrane conductance regulator gene. Hum Genet 1992; 88: 356.

53. Audrézet MP, Costes B, Ghanem N, et al. Screening for cystic fibrosis in dried blood spots of newborns. Molecular and Cellular Probes 1993; 7 : 101-6.

54. Williamson $R$. Universal community carrier screening for cystic fibrosis? Nature Genet 1993; 3 : 195-201.

55. Mennie ME, Gilfillan A, Compton $\mathbf{M}$, et al. Prenatal screening for cystic fibrosis. Lancet 1992 ; 340 : 214-6.

56. Brambati B, Tului L, Fattore S, Férec C. First trimester fetal screening of cystic fibrosis in a low-risk population. Lancet 1993 ; 342,8871 : 624.

57. White MB, Leppert $M$, Nielsen D, Zielenski J, Gerrard B, Stewart C, Dean M. $\mathrm{A}$ de novo cystic fibrosis mutation: CGA (Arg) to TGA (Stop) at codon 851 of the CFTR gene. Genomics 1991; 11 : 778-9.
(6\% des stérilités masculines) était héréditaire, de transmission autosomique récessive. La mise en évidence dans la série lilloise de huit sujets hétérozygotes $\Delta \mathrm{F} 508$ parmi dix-huit patients azoospermiques, suivie de l'observation, au moins dans leur série, de quelques sujets portant la mutation faux sens R117H [47], conforte l'hypothèse évoquée dès 1968 que les patients atteints d'agénésie déférentielle pouvaient avoir une forme très bénigne à expression uniquement génitale de mucoviscidose.

Il a maintenant été confirmé par différents groupes que $50 \%$ à $60 \%$ des patients agénésiques sont hétérozygotes $\Delta \mathrm{F} 508$ et qu'environ $10 \%$ d'entre eux sont hétérozygotes composites. Ces patients sont asymptomatiques au niveau respiratoire ou digestif; le test de la sueur est normal chez ces sujets, tout au plus retrouve-t-on assez souvent des valeurs de [Cl-] de 40 à $50 \mathrm{mEq} / 1$, c'est-à-dire discrètement supérieures à celles observées chez les sujets normaux (en général entre 20 et $30 \mathrm{mEq} / \mathrm{l})$. Au total aujourd'hui, si le CFTR est clairement impliqué dans cette maladie, d'importantes questions sont soulevées mais non réglées. Existe-t-il une hétérogénéité génétique qui impliquerait d'autres gènes que le CFTR chez les sujets stériles par agénésie des déférents? N'y aurait-il pas des mutations situées à l'extérieur de la région codante du gène CFTR - mutations introniques, mutations situées dans le promoteur - pouvant influencer les sites de régulation du gène? Doit-on aujourd'hui considérer l'agénésie déférentielle comme un "phénotype génital " de mucoviscidose ? Quoi qu'il en soit, les conséquences pratiques de la définition moléculaire de ce syndrome sont importantes et impliquent que l'on propose, dès à présent, aux sujets apparentés à ces patients ainsi qu'à leur partenaire (en cas de fécondation in vitro par ponction épididymaire) une recherche des mutations fréquentes du gène CFTR

\section{Les formes frustes}

L'analyse systématique du gène chez des patients adultes porteurs d'une dilatation des bronches et dont le test de la sueur était normal a permis également de montrer un excès d'hétérozygotes $\Delta \mathrm{F} 508$ et d'identifier clairement quelques hétérozygotes composites [48]. Ces formes de mucoviscidose a minima n'impliquent très probablement pas une mutation particulière du gène mais doivent correspondre à un discret dysfonctionnement de la protéine CFTR. Ainsi, la mutation G551S a été décrite chez deux sœurs présentant des valeurs à la limite de la normale pour un test de la sueur, associées à une forme très modérée de la maladie, d'expression uniquement pulmonaire [49]. Le groupe de Kerem a également rapporté récemment, dans une série de quinze patients, l'association de formes modérées de mucoviscidose à la mutation intronique $3849+10 \mathrm{~kb}$ (intron 19) sur un allèle (33\% de ces malades avaient un test de la sueur normal) [50].

\section{De la connaissance du gène aux applications cliniques}

\section{Diagnostic prénatal}

La connaissance de la plupart, sinon de toutes, les anomalies moléculaires du gène dans certaines populations permet de proposer, aux couples à risque d'avoir un enfant atteint, un diagnostic anténatal précoce et fiable à onze semaines d'aménorrhée. La technique de l'électrophorèse en gradient de gel dénaturant permet un choix spécifique des régions du gène à analyser, améliore la performance diagnostique et peut être modulée et adaptée selon l'origine des différentes populations [51]. La connaissance actuelle des principales mutations présentes dans un pays ou une région donnés permet de mettre en place des systèmes analytiques plus souples ou plus simples, comme par exemple l'amplification spécifique d'allèles ou le dot blot reverse* qui permettent très rapidement de rechercher jusqu'à trente mutations

\footnotetext{
* Dot blot reverse: hybridation du produit d'amplification, marqué par exemple par la biotine, à un filtre où l'on a fixé une batterie de sondes (oligonucléotides) correspondant aux différents allèles de la région amplifiée.
} 
différentes. Grâce à l'étude familiale des polymorphismes de type microsatellite, il est possible de proposer un diagnostic lorsque les mutations ne sont pas identifiées, comme par exemple dans les populations d'Europe du Nord où près de $10 \%$ des allèles CFTR n'ont pas été caractérisés. Mais surtout, au sein des populations du sud de l'Europe où $30 \%$ des allèles CFTR sont non identifiés, le diagnostic repose sur la mise en évidence de marqueurs intragéniques et nécessite de pouvoir disposer d'un prélèvement de l'enfant atteint [52].

\section{Diagnostic néonatal}

L'analyse directe des mutations du gène, de la mutation $\Delta \mathrm{F} 508$ mais également des autres mutations non rares, a permis de mettre en place dans certaines régions un test de dépistage néonatal en une seule étape couplant le dosage de la trypsine immunoréactive à la recherche de mutations [53]. Le dépistage de la maladie a lieu dans les premières semaines de vie des nouveau-nés atteints, évitant ainsi le fort taux de rappel qui était observé lors de l'utilisation de la seule trypsine immunoréactive.

\section{Diagnostic des porteurs}

Le problème du dépistage des hétérozygotes dans la population générale a été un sujet largement débattu au sein des diverses sociétés de génétique [54]. La recherche des mutations fréquentes permet de dépister $80 \%$ à $90 \%$ des mutations dans beaucoup de pays d'Europe et aux États-Unis et, bien que cette sensibilité ne soit pas parfaite, ces résultats ont conduit à la mise en place d'expériences pilotes de dépistage des hétérozygotes en Angleterre et au Danemark. Différentes stratégies ont été proposées, par exemple la recherche des cinq mutations les plus fréquentes chez les femmes en début de grossesse [55] ou l'analyse des mutations les plus fréquentes chez les jeunes couples avant toute grossesse dans les régions à risque. Une recherche systématique de mutations fréquentes a même été réalisée en Italie à partir des cellules foetales obtenues par prélève$\mathrm{m} / \mathrm{s} n^{\circ} 6-7$ vol. 10, juin-juillet 94 ment de liquide amniotique réalisé en vue du diagnostic caryotypique [56]. Tout cela exige la mise en place de structures de prise en charge médicale spécialisée, de consultations de conseil génétique en particulier, pour qu'une information claire et détaillée puisse être offerte aux sujets dépistés.

Les résultats de ces diverses expériences et les perspectives qu'ils ouvrent vont devoir être largement débattus; les impératifs socioéconomiques et éthiques devront être pris en compte avant que ces études pilotes ne soient généralisables.

\section{Conclusion}

Plus de quatre cents mutations dans le gène CFTR ont été rapportées depuis quatre ans et ce nombre sera très probablement dépassé dans les prochains mois. A côté d'une mutation fréquente, probablement ancestrale, la délétion $\Delta \mathrm{F} 508$, seules quatre ou cinq autres ont été retrouvées dans les différentes populations à une fréquence supérieure à $1 \%$. La très grande majorité des autres anomalies moléculaires sont des mutations privées, présentes sur quelquesuns des chromosomes examinés. Pour l'essentiel, il s'agit d'anomalies ponctuelles conduisant à des mutations faux sens ou non sens, ou à de courtes insertions/délétions; les délétions de taille moyenne ou de grande taille sont exceptionnelles. Exceptionnelles semblent également les néo-mutations : à ce jour il n'en a été rapporté qu'une seule [57]. Les mutations connues peuvent être regroupées en au moins quatre classes distinctes qui permettent de rassembler les allèles $C F T R$ mutés conduisant à des dysfonctionnements au niveau du canal chlorure. Il est ainsi possible de mieux étayer les relations phénotype/génotype montrant à l'évidence que certains signes cliniques, telles la suffisance pancréatique ou les formes cliniques de sévérité modérée observée chez des adultes, sont génétiquement déterminés. Cela permet aussi d'augurer pour l'avenir une approche thérapeutique différente selon le génotype des patients. Ainsi, à côté de la thérapie génique $\left(m / s n^{\circ} 1\right.$, vol. 7, p. 84) aux États-Unis, en particulier, avec les premiers essais chez l'homme de transfert du gène $C F T R$ à l'aide d'adénovirus génétiquement modifiés, la place pour une approche pharmacologique est très largement ouverte pour certains patients, en particulier ceux qui présentent des mutations conduisant à des troubles de la conduction canalaire. Il suffit de se rappeler qu'en 1985 on ignorait la localisation chromosomique du gène de la mucoviscidose pour apprécier les progrès réalisés depuis huit ans et mesurer combien l'étude du gène CFTR est un modèle d'étude de maladie monogénique chez l'homme

\section{Remerciements}

Les auteurs remercient l'Association française de lutte contre la mucoviscidose pour son soutien financier.

\section{Summary}

Cystic fibrosis gene mutations

The gene responsible for cystic fibrosis was cloned in 1989 and extensive analyses have been performed since showing that the cystic fibrosis transmembrane conductance regulator (CFTR) is a chloride channel regulated by a cAMP dependent phosphorylation. The identification of more than 400 different mutated alleles shows now a precise distribution of these mutations in the gene which varies with geographic origin of patients. These molecular analyses have permitted genotype/phenotype correlation to be established and it is evident that some clinical symptoms are genetically determined. The precise characterization of gene defects has led to a better knowledge of CFTR's channel function and to a better understanding of how CF associated mutations disrupt CFTR function. 